'Sub Departamento de Medicina, Hospital Base de Valdivia e Instituto de Medicina, Universidad Austral de Chile. Valdivia, Chile.

2Programa de Control y Eliminación Tuberculosis, Ministerio de Salud. Chile. ${ }^{3}$ Centro de Epidemiología y Políticas de Salud, Universidad del Desarrollo. Santiago, Chile.

Trabajo no recibió financiamiento. Los autores declaran no tener conflictos de interés.

Recibido el 10 de septiembre de 2018, aceptado el 18 de junio de 2019.

Correspondencia a: Dr. Alberto Fica Sub Departamento de Medicina, Hospital Base de Valdivia, Chile. Bueras 1003. Valdivia, Chile. albertoficacubillos@gmail.com

\title{
El deterioro de la tuberculosis en Chile
}

\author{
ALBERTO FICA ${ }^{1}$, TANIA HERRERA ${ }^{2}$, XIMENA AGUILERA ${ }^{3}$
}

\section{Worsening situation of tuberculosis in Chile}

Background: Recent data suggest an increase in tuberculosis (TB) incidence in Chile. Aim: To evaluate recent epidemiological trends, geographic extension and potential factors associated with TB reemergence in Chile. Material and Methods: Data analysis from official sources and trend analysis. Results: TB incidence rate increased from 12.3 (2014) to 14.7 (2017) per 100,000 inhabitants. Morbidity rates also increased in nine out of 15 regions. The proportion of TB cases in specific groups has also increased in the last six years: HIV/AIDS (68\%), immigrants (118\%), drug users/alcoholics (267\%) and homeless people (370\%). Several indicators of the national TB program performance have deteriorated including TB case detection, HIV co-infection study and contact tracing activities. Overall results indicate a higher than expected case-fatality ratio $(>3 \%)$, high rates of loss from follow-up (>5\%), and low percentage of cohort healing rate $(<90 \%)$. This decline is associated with a Control Program with scarce human resources whose central budget decreased by $90 \%$ from 2008 to 2014. New molecular diagnostic tools and liquid media culture were only recently implemented. Conclusions: TB trends and overall program performance indicators have deteriorated in recent years in Chile and several factors appear to be involved. Multiple strategies will be required to rectify this situation.

(Rev Med Chile 2019; 147: 1042-1052)

Key words: Emigrants and Immigrants; Financial Support; HIV; Tuberculosis.

$\mathrm{L}$ as tasas de tuberculosis (TB) han disminuido a nivel mundial gracias al desarrollo humano, un indicador que conjuga el ingreso per capita, la expectativa de vida y el nivel educacional e intervenciones como la vacuna BCG, el tamizaje de sintomáticos respiratorios, el estudio de contactos, el tratamiento de infección latente en pacientes de alto riesgo, la gratuidad y control del tratamiento ${ }^{1-9}$.

El control de la TB requiere un alto compromiso político, apoyo financiero, recursos humanos e infraestructura de laboratorio y su expansión presupuestaria mejora los resultados ${ }^{10,11}$. En contraste, el recorte financiero, genera aumentos en la morbilidad y mortalidad, como sucedió en diversos lugares durante los años 70-80 $0^{12,13}$. El déficit financiero, el desmantelamiento de los servicios de salud, la migración humana, el aumento de la pobreza y la pandemia de VIH/SIDA, estuvieron asociados a la resurgencia de la TB a nivel mundial y una situación de emergencia fue declarada ${ }^{14}$. Este problema empeoró con la expansión de cepas multidrogoresistentes (MDR) y cepas con resistencia extendida (XDR).

En Chile, la TB venía declinando progresivamente desde el siglo pasado. La mortalidad bajó desde 250 a 1,5 defunciones por 100.000 habitantes (1947-2010) $)^{9,15}$ y la morbilidad se redujo desde 386 a 13 casos por $100.000(1949-2010)^{15,16}$. Este logro estuvo estrechamente asociado a la mejoría en las condiciones de vida ${ }^{17}$. La creación del Servicio Nacional de Salud (1952) junto al inicio de la terapia con estreptomicina-PAS (1948) y las campañas de vacunación masiva con la vacuna BCG (1951) facilitaron el control de la TB en Chile $^{18}$. En contraste se mantuvo una alta carga de enfermedad en países vecinos como Perú y Bolivia $^{19-23}$. 
El último informe oficial sobre TB muestra un aumento de casos en Chile, después de un largo período de estancamiento ${ }^{24}$. Este retroceso sucede a pesar del desarrollo económico, que nos sitúa como miembro pleno de la Organización para la Cooperación y Desarrollo Económico (OCDE) con altos ingresos. El objetivo de este trabajo es analizar la situación actual de la TB en Chile, su tendencia y explorar las posibles causas de su resurgencia.

\section{Material y Métodos}

Estudio descriptivo que revisa la situación epidemiológica sobre TB en población general y en grupos especiales, explorando su asociación con determinantes sociales y otros factores, relacionados a los recursos y desempeño del Programa de Control de la TB en Chile (PROCET). Los datos fueron extraídos de diferentes fuentes mencionadas en el texto según aparición. Específicamente, se analizaron factores socioeconómicos (proporción de personas bajo la línea de pobreza según ingreso, pobreza multidimensional y coeficiente de Gini). Se analizaron también las tendencias de los grupos de riesgo registradas al momento del diagnóstico (como proporción del total) tales como VIH/SIDA, pueblos originarios, usuarios de drogas ilegales o alcohólicos, edad mayor, situación de calle, personal de salud o personas privadas de libertad (PPL). Para la TB asociada a cepas multidrogoresistentes (MDR) y extensivamente resistentes (XDR), se extrajo el número de estos aislamientos desde los informes oficiales y su presencia contabilizada en forma simple. Los datos del presupuesto del PROCET fueron obtenidos desde el Ministerio de Salud bajo la Ley de Transparencia. Sus valores fueron actualizados al 2014 y transformados a dólares americanos (US\$). Este presupuesto no incluye los gastos efectuados en forma regional o municipal destinados a actividades relacionadas a la TB. Para el análisis de las características operacionales del PROCET, dotación de RRHH y cobertura vacuna BCG, se extrajeron los datos respectivos de los informes oficiales.

Para las variables coeficiente de Gini, pobreza, pobreza multidimensional, proporción de pacientes con TB pertenecientes a diferentes subgrupos de riesgo, tasa de pacientes con infec- ción VIH/SIDA por regiones y presupuesto anual del PROCET, las tendencias en el tiempo fueron analizadas con el coeficiente de Spearman, una prueba no paramétrica con un rango de $-1,00$ a $+1,00$. EL nivel de significación estadística usado fue $\mathrm{p}<0,05$. Las variables operativas del PROCET fueron analizadas comparativamente en forma cualitativa respecto a los estándares establecidos por el propio Programa.

\section{Resultados}

\section{Tendencias epidemiológicas}

La tendencia de la incidencia de TB (todas sus formas) desde 1990 muestra 3 etapas (Figura 1), primero una declinación prolongada que dura 15 años (1990-2005), seguido por un estancamiento (2006-2014) y finalmente, una etapa de reemergencia epidemiológica (12,3 el 2014 a 14,7 por 100.000 habs. el 2017) ${ }^{15,24-27}$. El aumento de la morbilidad se observa en 9 de las 15 regiones del país (Figura 2). El 2017, tres regiones del norte tenían tasas sobre 20 casos por 100.000 habs, el umbral de eliminación (Figura 2).

\section{Factores socioeconómicos}

Los porcentajes de pobreza poblacional, la pobreza multidimensional (PMD) y coeficiente de Gini muestran una mejoría continua ( $\mathrm{r}-1,00$ para cada uno de ellos) (Figura 3$)^{27-29}$. En 2 de las 8 regiones con aumento de TB hubo un incremento reciente de la pobreza (II Región) y de la PMD (I y IV Región; datos no mostrados).

\section{Grupos de riesgo}

Los extranjeros incrementaron significativamente su importancia relativa en el total de casos entre el 2012 y el 2017 (118\% de incremento, $r+0,96 ; p<0,05)$, mientras otros grupos mostraron una tendencia incremental aunque no significativa incluyendo pacientes con VIH/ SIDA $(5,7$ a $9,6 \% ;+68 \%)$, usuarios de drogas ilegales o alcohólicos (267\%) y en situación de calle $(+370 \%)$ (Figura 4$)^{15,24-27,31,32}$. En la Tabla 1 se indica el porcentaje de pacientes con TB el año 2017 pertenecientes a diferentes grupos según región. Las zonas del norte parecen estar afectadas por una importante contribución de casos entre extranjeros. (10,0-31,1\%), pueblos originarios (hasta $31 \%$ ) y usuarios de drogas ilegales (hasta 

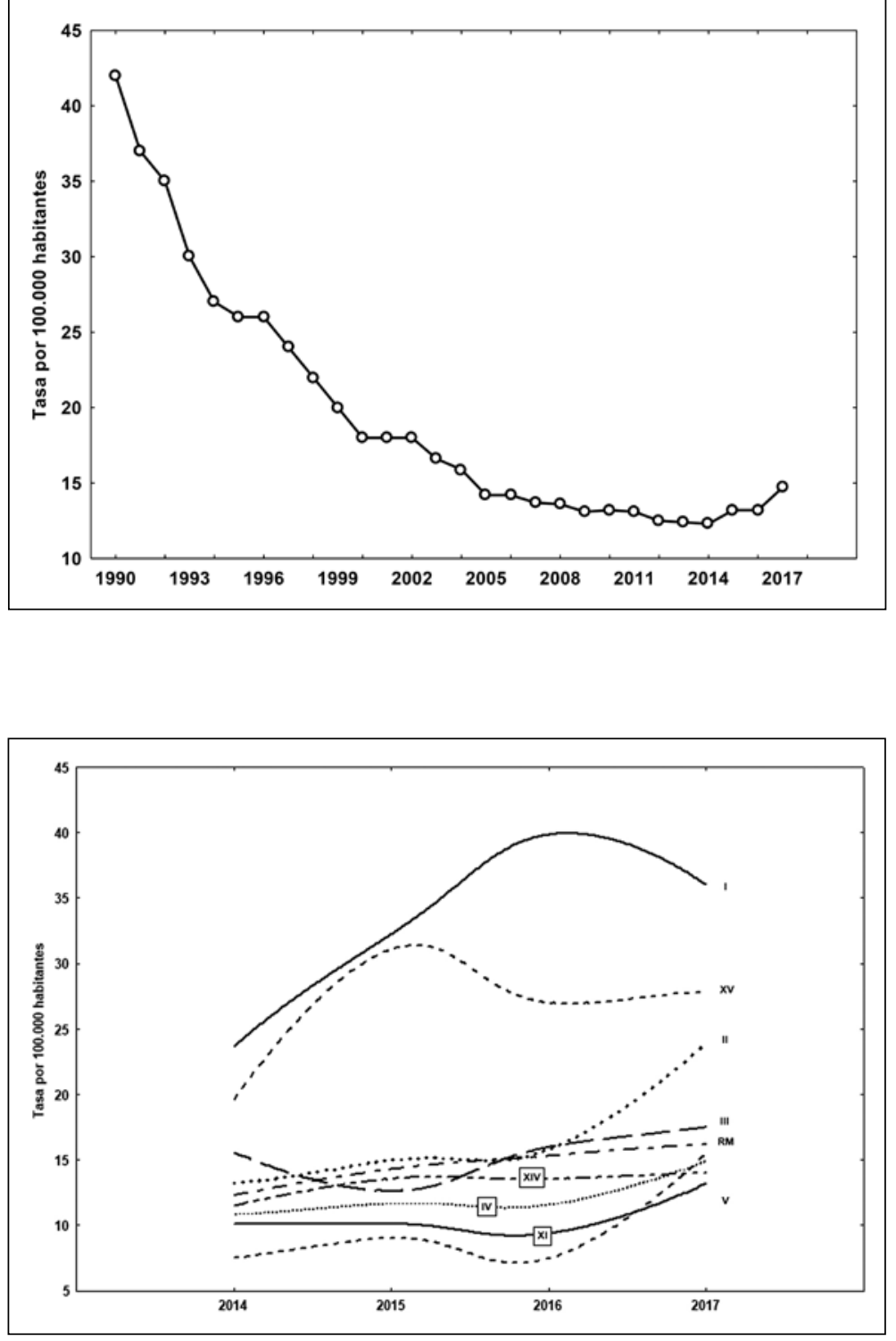

Figura 1. Tasa de incidencia de Tuberculosis en Chile. Todas sus formas, 1990-2017.

Figura 2. Resurgencia de la TB en 9 regiones del país según datos de morbilidad en todas sus formas. El deterioro epidemiológico de la TB está ocurriendo en varias zonas del país. Las 9 regiones indicadas en la figura muestran una tendencia al aumento con coeficientes de correlación de Spearman positivos al considerar los 4 últimos años (20142017). Los coeficientes por regiones fueron I: 0,80; XV:0,40; II: 1,00; III: 0,80; IV: 0,80; V: 0,50; RM: 1,00; XI: 0,40 y XIV: 0,80 . Las regiones I y $X V$ están en el norte del país. Las curvas fueron ajustadas por el método de los mínimos cuadrados para facilitar su lectura gráfica.

$16 \%)$. Las zonas del sur están observando la participación de pacientes en situación de calle (25\%), alcohólicos y pacientes con diabetes mellitus. La TB en pacientes con VIH/SIDA es transversal y la Región Metropolitana muestra varios grupos de riesgo en forma simultánea.

Hacia el año 2014, cerca de 2,3\% de la población total de Chile estaba constituida por inmigrantes especialmente en el norte $(7,4 \%$ en la I Región y 5,8\% en la XV región) ${ }^{33}$. El año 2013, la comunidad de inmigrantes de Perú representaban el grupo más importante y la proveniente de Bolivia, el cuarto grupo en importancia, con la mayor parte de ellos en el norte ${ }^{33}$. De acuerdo a datos oficiales, los inmigrantes tienen valores superiores de PMD (25,2\% versus $20,3 \%)$ por peores valores en hacinamiento ${ }^{34}$.

Las infecciones por VIH aumentaron 36\% entre el 2010-2016 ${ }^{34}$ y la incidencia escaló de 21,4 a 28,9 por 100.000 habitantes $\geq 13$ años entre el 

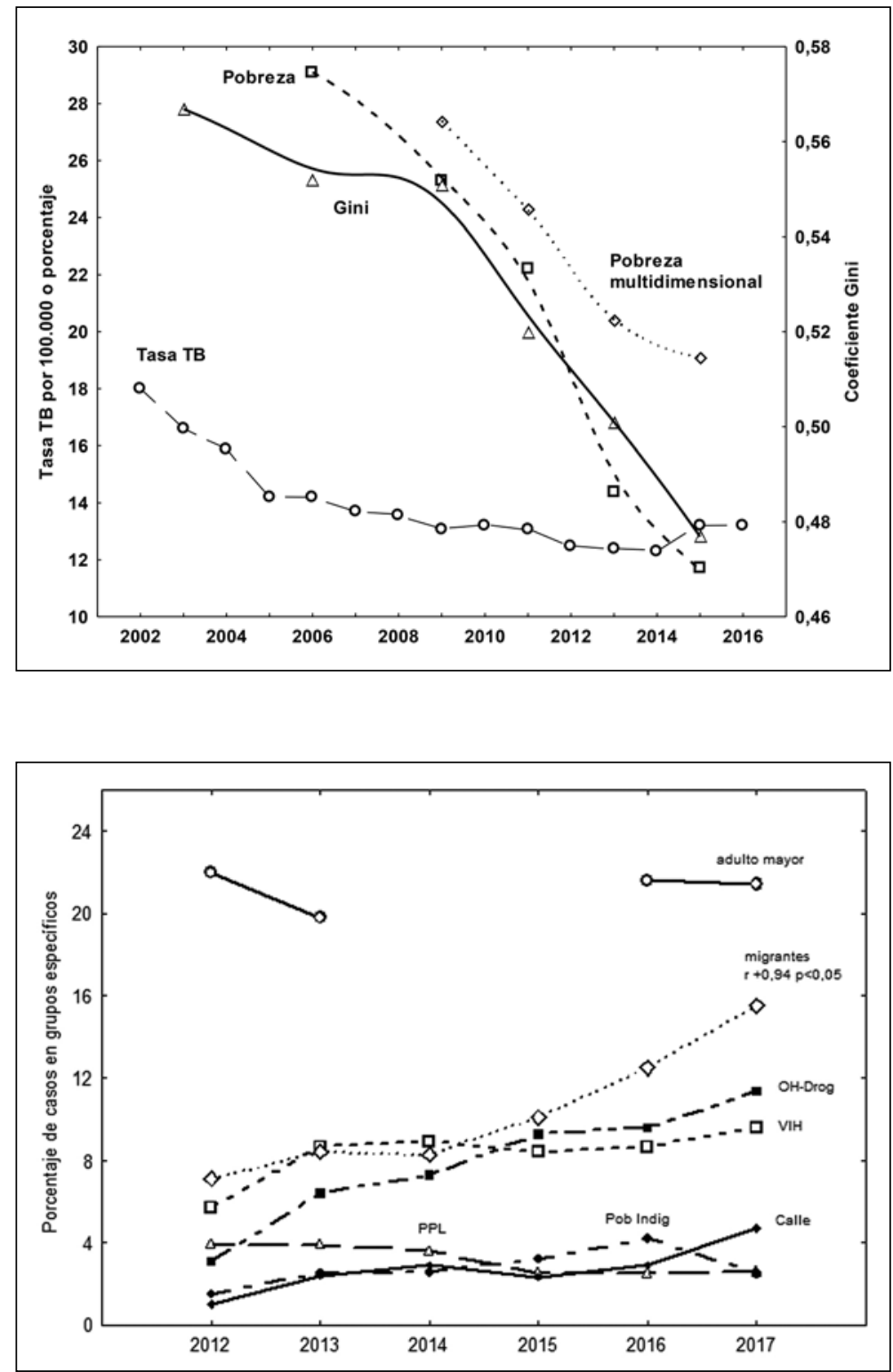

Figura 3. Incidencia de TB y parámetros socioeconómicos, Chile 2001-2016. A nivel nacional, el porcentaje de pobreza, de pobreza multidimensional y el coeficiente de Gini han mejorado durante el estancamiento epidemiológico de la TB en Chile. La PMD es un indicador que integra información sobre desempleo, escolaridad, salud, seguro social, pensión de vejez, vivienda, hacinamiento y estado nutricional. El coeficiente de Gini mide inequidad en la distribución del ingreso (Rango 0 [no inequidad] a 1 [inequidad total]).

Figura 4. $T B$ en diferentes grupos de riesgo en años recientes. Análisis por coeficiente de correlación de Spearman entre los años 2012 y 2017. Los porcentajes de pacientes con TB pertenecientes a varios grupos específicos han aumentado en años recientes siendo significativo para el grupo inmigrantes ( $p<0,05)$. $\mathrm{OH}$-Drog: alcohólicos y drogadictos; Pob Indig: pueblos originarios; PPL: personas privadas de libertad; Calle: personas en situación de calle.

2010 y el $2015^{36}$. Cuarenta y dos coma cuatro por ciento de los pacientes que inician terapia antiviral tienen recuentos CD $4 \leq 200 / \mu \mathrm{L}$ y $22 \%$ es diagnosticado en etapa $\mathrm{C}^{37}$, subrayando una epidemia descontrolada y un diagnóstico tardío posiblemente porque la mayor parte del presupuesto se gasta en terapia (95\%) y no en prevención con un diagnóstico sin acercamiento a la comunidad ni actividades para alcanzar grupos de alto ries$\mathrm{go}^{38,39}$. Según nuestros cálculos, la incidencia de
VIH/SIDA en $\geq 13$ años entre el 2010 y el 2015 aumentó significativamente en 6 de las 15 regiones de Chile incluyendo 2 de aquellas con un aumento de TB (Regiones I y II) y las 9 regiones restantes mostraron una tendencia al aumento ${ }^{36}$ lo que sugiere un rol sobre la TB. Aunque el estudio de co-infección VIH/TB ha mejorado en los últimos años (69\% de cobertura el 2016), 2 regiones con elevada incidencia de TB tienen bajos porcentajes de estudio $(<50 \% \text {, regiones I y II })^{24}$. 
Tabla 1. Porcentaje de diferentes grupos de riesgo en pacientes con TB diagnosticados el 2017 en regiones seleccionadas (Tabla adaptada de referencia 27 )

\begin{tabular}{|c|c|c|c|c|c|c|c|c|}
\hline $\begin{array}{l}\text { Región/ } \\
\text { Zona }\end{array}$ & VIH/SIDA & $\begin{array}{c}\text { Inmi- } \\
\text { grantes }\end{array}$ & PPL & $\begin{array}{l}\text { Pueblos } \\
\text { originarios }\end{array}$ & $\begin{array}{l}\text { Situación } \\
\text { de calle }\end{array}$ & $\begin{array}{l}\text { Alcohóli- } \\
\text { cos }\end{array}$ & $\begin{array}{l}\text { Droga- } \\
\text { dictos }\end{array}$ & $\begin{array}{l}\text { Diabetes } \\
\text { mellitus }\end{array}$ \\
\hline \multicolumn{9}{|l|}{ Norte } \\
\hline 1 & $9,2 \%$ & $31,1 \%$ & $2,5 \%$ & $9,2 \%$ & $5,0 \%$ & $10,1 \%$ & $10,9 \%$ & $7,6 \%$ \\
\hline$X V$ & $12,7 \%$ & $17,5 \%$ & $1,6 \%$ & $31,1 \%$ & $1,6 \%$ & $7,9 \%$ & $7,9 \%$ & $1,6 \%$ \\
\hline ॥ & $9,7 \%$ & $23,4 \%$ & $3,4 \%$ & $1,4 \%$ & $3,4 \%$ & $11,0 \%$ & $15,9 \%$ & $13,8 \%$ \\
\hline III & $8,0 \%$ & $10,0 \%$ & $2,0 \%$ & $0,0 \%$ & $10,0 \%$ & $10,0 \%$ & $10,0 \%$ & $4,0 \%$ \\
\hline \multicolumn{9}{|l|}{ Central } \\
\hline RM & $12,5 \%$ & $25,3 \%$ & $1,5 \%$ & $2,0 \%$ & $4,7 \%$ & $12,0 \%$ & $12,9 \%$ & $8,0 \%$ \\
\hline \multicolumn{9}{|l|}{ Sur } \\
\hline$X I$ & $6,3 \%$ & $6,3 \%$ & $0,0 \%$ & $0,0 \%$ & $37,5 \%$ & $37,5 \%$ & $0,0 \%$ & $6,3 \%$ \\
\hline XII & $8,0 \%$ & $8,0 \%$ & $4,0 \%$ & $4,0 \%$ & $0,0 \%$ & $12,0 \%$ & $0,0 \%$ & $12,0 \%$ \\
\hline
\end{tabular}

RM: Región Metropolitana. Los porcentajes relevantes se muestran en negrita.

\section{Emergencia de aislados MDR y XDR}

Estudios de prevalencia cada 3 a 5 años indican que sólo en los años 1981 y 2001 se registraron cepas MDR en pacientes nunca antes tratados con prevalencias de 0,2 y $0,7 \%$ (3 y 6 cepas, respectivamente $)^{40}$. En años recientes han aumentado las cepas MDR en estos pacientes (18 sólo el año $2017)^{24,27,32}$. Casos con cepas XDR han sido detectados en 2011, 2013 y nuevamente en 2016 (1-2 casos por año) $)^{21,24,26}$.

\section{Problemas financieros y operacionales}

El PROCET requiere actividades locales y nacionales. Los esfuerzos locales se realizan en los centros de atención primaria, que dependen principalmente de las municipalidades quienes determinan los RRHH disponibles, insumos, procedimientos de pesquisa, estudio de contactos y tratamiento de pacientes con TB activa o infección latente. Estas dependencias reciben un presupuesto anual desde el gobierno de acuerdo al número de personas inscritas pero no tienen posibilidades de obtener más fondos en casos de contingencia y depende de la prioridad que otorguen las autoridades locales. Desafortunadamente, la información sobre el presupuesto anual municipal o por Servicios de Salud destinado al control de la TB a nivel local es difícil de obtener. No obstante, las acciones nacionales que incluyen aspectos regulatorios, educacionales y de modernización de laboratorios, dependen de un presupuesto fijo central. El presupuesto global de salud de Chile aumentó 2,63 veces entre el 2003 y el 2014, alcanzando cerca de los 16 mil millones de dólares el último año analizado (Figura 5) y los fondos destinados a Salud Pública que incluye al PROCET aumentaron 2,88 veces. El del PROCET fue recortado durante la primera década del siglo XXI, desde aproximadamente 1 millón US\$ anuales el 2000-2001 a dos tercios de este valor el 2006 (Figura 6) luego de la reforma de salud de 2005 que modificó la autoridad sanitaria. Este cambio desmanteló los equipos locales de TB, limitó la modernización de laboratorios, labores de supervisión y la capacitación y entrenamiento del personal ${ }^{41}$. El presupuesto central del PROCET fue reasignado a sus valores previos en los años 2007-2008, pero fue nuevamente recortado a un décimo de su valor original el 2009 (Figura 6). A pesar de la intención de traspasar el recorte a los Servicios de Salud, estos no pudieron destinar recursos especiales para actividades relacionadas al programa de la TB. Las decisiones de recorte presupuestario, realizadas durante gobiernos de centro-izquierda, que habían definido a la TB como prioridad sanitaria, motivaron la renuncia del encargado del Programa. Este segundo recorte hizo difícil sostener muchas de las actividades del PROCET.

El año 2012, una auditoría externa desarrollada al PROCET por la Organización Panamericana de la Salud (OPS), identificó problemas graves y un desempeño del programa bajo los estándares internacionales. Entre ellos se constató la pérdida de prioridad reflejada en las reducciones financie- 


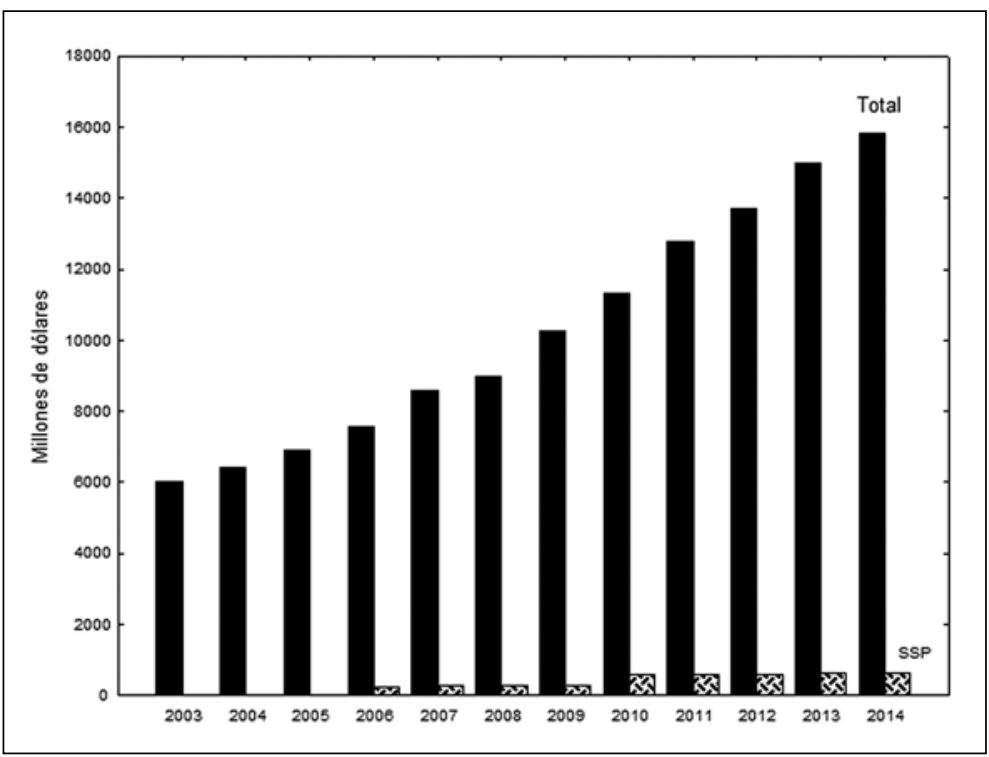

Figura 5. Evolución del gasto en salud Chile 2003-2014 en millones de dólares. El presupuesto de salud ha aumentado en forma constante en los últimos años, incluyendo el de la subsecretaría de Salud Pública (SSP). El presupuesto destinado a Salud Pública en tamaño es minúsculo (cerca del 4\%, 655 millones US\$ para el 2014) al compararse con el presupuesto global. Datos actualizados al 2014 (1 US\$ = 552,98 CLP).

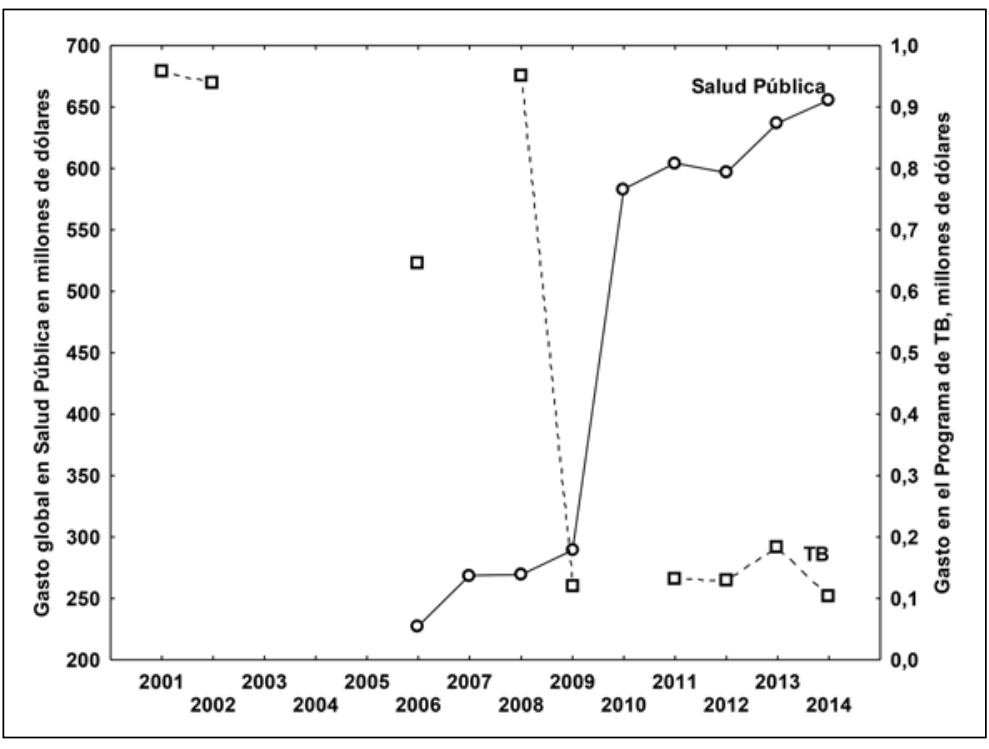

Figura 6. Gasto en el presupuesto de Salud Pública y en el Programa de Control de la TB (PROCET), Chile 2001-2014. Datos actualizados a diciembre de 2014 (1 US\$ $=552,98 \mathrm{CLP}$ ). Varios recortes presupuestarios han sido aplicados al PROCET (2006, 2009 y 2014). ras y de RRHH, con equipos locales incompletos con tiempo insuficiente, perjuicio en funciones operativas, como la pesquisa de sintomáticos respiratorios, y su concentración excesiva en niveles secundarios y no primarios de atención. Además se detectó bajas tasas de curación comparadas con el estándar establecido $(\geq 90 \%)$, alta pérdida de pacientes en seguimiento $(>5 \%$ según lo aceptable por el estándar local) y alta letalidad (> 3\% establecido como referente). La detección de VIH entre los pacientes con TB era insuficiente, al igual que los registros de pacientes VIH/SIDA que recibían terapia para infección latente. La escena se complementaba con un bajo porcentaje de contactos estudiados (estándar > 90\%), problemas no resueltos en prisiones, insumos y 
provisión de medicamentos y una infraestructura de laboratorios anticuada ${ }^{42}$. Estos problemas fueron publicados y comunicados a las autoridades de salud el año 2013, pero el presupuesto central fue nuevamente recortado por tercera vez al año siguiente (Figura 6) y en esta ocasión bajo el mandato de un gobierno de centro-derecha. Entre el 2001 y el 2014 el presupuesto central fue recortado significativamente $(\mathrm{r}-0,80, \mathrm{p}<0,05)$. La misma autoridad sanitaria multó a algunas reparticiones por deficiencias que ponían en riesgo al personal de salud y retrasaban el diagnóstico (Hospital San Borja Arriarán en la Región Metropolitana) ${ }^{43}$.

Luego de gestiones efectuadas por Sociedades Científicas junto a los comentarios vertidos por la auditoría de la OPS ante las autoridades de salud el 2015, se logró un compromiso para aumentar el presupuesto del programa de TB a través de la asignación fondos hacia los servicios de salud desde el año 2015 (Ítem 22), el que permite gastos operacionales (insumos, medicamentos, equipos menores, mantención de equipos, fiscalización en terreno o capacitación) pero no la contratación de RHHH. Los montos asignados por esta vía fueron superiores al presupuesto histórico $(<1$ millón US\$), llegando a los 3,6 millones de dólares el 2015 y 2016 (datos provistos por PROCET) lo que ha permitido mantener parte de los aspectos operativos y una modernización de los laboratorios.

Los niveles intermedios del Programa de TB tienen deficiencia de personal con contratos dependientes de los Servicios de Salud locales y no del nivel central. Hacia el 2011, había un déficit de $72,7 \%$ de las horas médicas semanales requeridas por el programa, para horas de enfermería era de $61,6 \%$ y para tecnólogos médicos de $65,3 \%{ }^{25}$, fenómenos posiblemente asociados a la baja importancia asignada por las autoridades locales al programa de TB.

Se estima que se deben efectuar aproximadamente 50 tamizajes cada 1.000 visitas médicas de pacientes adultos (índice de pesquisa) para detectar casos bacilíferos y cortar la cadena de transmisión. Entre el 2006 y el 2010, las muestras respiratorias para estudio de $\mathrm{TB}$ disminuyeron de 310.690 a 241.486 anuales, con un índice de pesquisa $<50$ por 1.000 visitas en todas las regiones $^{44}$. Actualmente se mantiene este bajo índice de pesquisa en todas las regiones, excepto en una $^{24}$. Sólo recién el 2015, el número anual de exámenes alcanzó lo observado una década antes ${ }^{24}$. Esta baja pesquisa es otro factor que contribuye al retroceso epidemiológico.

Las deficiencias detectadas por la visita de la OPS siguen presentes. Por ejemplo, la tasa de curación para la cohorte del 2012 fue de $80,6 \%$ y de $72 \%$ el 2015 (estándar $\geq 90 \%$ ), la proporción de pacientes perdidos durante el seguimiento empeoró de 6,5\% a 8,6\% entre el 2012 y el 2015 (estándar $\leq 5 \%$ ) y la letalidad alcanzó 9,5\% y luego $11,4 \%$ en el mismo período (estándar $\leq 3 \%$ ) ${ }^{26}$.

\section{Vacuna BCG}

Con oscilaciones, las coberturas de la vacuna BCG en recién nacidos han permanecido adecuadas en el tiempo y no parecen tener un rol en la situación reciente de la TB en Chile ${ }^{24,45}$.

\section{Laboratorio de TB}

Hasta el año 2016, el diagnóstico de TB en Chile en el sistema público (que atiende a $80 \%$ de la población), estaba mayoritariamente basado en métodos convencionales como la tinción de Zielh-Neelsen, cultivos en medios sólidos y radiografías. Además, los estudios de susceptibilidad no fueron universales hasta el $2014 \mathrm{y}$ antes de ello, restringidos a pacientes de alto riesgo (inmunosuprimidos, abandonos, antes tratados, etc.). Desde el 2017, los cultivos en medios líquidos y los equipos Xpert MTB/RIF han sido incorporados progresivamente en la red de diagnóstico del sector público, pero no al nivel primario. Este salto es relevante, ya que ambas tecnologías mejoran y adelantan el diagnóstico de TB activa. Por ejemplo, la inclusión de Xpert MTB/RIF aumentó la sensibilidad diagnóstica desde $66 \%$ a $91 \%$ en muestras respiratorias en pacientes con VIH y TB y la detección de casos con resistencia ${ }^{46}$.

\section{Discusión}

Después de décadas de un control sostenido, la TB en Chile está aumentando, fenómeno anticipado por una curva epidemiológica estancada. El aumento de la TB afecta a varias regiones del país y se combina con la presencia ocasional de cepas MDR y XDR, el incremento de los casos en pacientes con VIH/SIDA, en inmigrantes, en drogadictos o alcohólicos, en personas de pueblos originarios y en situación de calle. Este ascenso es precedido por un Programa de Control de 
la TB debilitado, diezmado y sometido a varios recortes presupuestarios en diferentes gobiernos a pesar de las recomendaciones de la OPS. Varios indicadores operativos del Programa chileno de TB demuestran defectos sustantivos incluyendo el tamizaje de sintomáticos respiratorios, el estudio de contactos de casos bacilíferos, tasas de letalidad y pérdidas de seguimiento elevadas y bajos porcentajes de curaciones de las cohortes tratadas. El escenario se oscurece por el estudio deficitario de la coinfección VIH y por el uso de técnicas diagnósticas desactualizadas, que sólo recién comienzan a modernizarse.

En contraste con el gran peso que tuvo la pobreza, desempleo, desnutrición y hacinamiento en la epidemiología de la TB en el pasado y en su resurgencia en los años 80 en Latinoamérica y USA $^{3,4,8,12,13,47}$, estos factores no parecen tener un rol transversal actualmente. Esto no significa, que todavía no sean importantes en su epidemiología sino que subrayan la relevancia de nuevos factores y situaciones en el riesgo de TB.

La TB entre inmigrantes es importante $y$ representa la mayor parte de los casos en países desarrollados. Por ejemplo, en USA; $66 \%$ de los casos registrados ocurre en pacientes nacidos en países extranjeros, una tasa que no disminuye en este grupo y que se concentra en zonas donde ellos entran o viven ${ }^{48}$. Las tasas de TB en Perú y Bolivia son aproximadamente 10 veces superiores a las observadas en Chile y una importante fracción de inmigrantes proviene de estos 2 países vecinos, con olas recientes dominadas por ciudadanos de Venezuela y Haitín ${ }^{33}$. Una fracción de ellos viven en condiciones de hacinamiento que es un factor de riesgo conocido para la transmisión de la $\mathrm{TB}^{3,4,8}$. Actualmente, no existe en Chile una política de tamizaje de algunas enfermedades para inmigrantes, como se aplica en USA o Europa y su mera implementación es difícil aún cuando su tratamiento es gratuito ${ }^{49-51}$.

La epidemia descontrolada de VIH en Chile parece contribuir al deterioro de la TB que explica en parte el aumento de la proporción de pacientes con VIH/SIDA con TB. Déficits en el estudio de coinfección VIH en los pacientes con TB y en el tratamiento de la infección latente también están contribuyendo a este fenómeno y señalan deficiencias del trabajo médico, aunque se ha observado una mejoría reciente en los indicadores respectivos. Estimaciones en la Región Metropolitana indican cifras de 380 por 100.000 habitantes de TB en pacientes $\mathrm{VIH}$, un riesgo relativo aproximado 35 veces superior a la población general ${ }^{52}$.

A pesar que el porcentaje de PPL en pacientes con TB no ha aumentado en los últimos años, la detección de casos depende de los esfuerzos locales que pueden variar entre los diferentes centros penitenciarios. Estudios recientes sobre TB en cárceles en Chile han demostrado una incidencia cerca de 8 veces superior a la población general ( 105 vs $\sim 12$ por 100.000 habs hacia el 2013) y en el caso de infección latente, una alta tasa de transmisión en prisiones ${ }^{53}$. Chile tiene una de las más altas tasas poblacionales de PPL dentro de los países de la OCDE con un hacinamiento crítico haciendo favorable la transmisión tanto de cepas susceptibles como resistentes de $M$. tuberculosis ${ }^{54-58}$.

Nuestro trabajo identificó también, dos fenómenos importantes: los recortes presupuestarios y una profunda y prolongada debilidad del PROCET en todo el país. Con la excepción de la cobertura de la vacuna BCG en los recién nacidos, cada componente estratégico de la lucha contra la TB está debilitado, incluyendo las acciones de tamizaje, tasas de curación, letalidad y seguimiento de las cohortes en tratamiento, el personal disponible y la infraestructura de laboratorio. Gobiernos consecutivos de diferente orientación política redujeron el presupuesto directo del PROCET, revelando una respuesta negligente mantenida y profundizada a pesar de las recomendaciones de corrección efectuadas por la OPS. Las tasas de TB han experimentado sólo un leve aumento promedio, lo que puede considerarse posible por el gran compromiso del personal que aún queda disponible. Si no se efectúan correcciones urgentes, el problema evolucionará a mayores tasas de TB con una expansión de casos asociados a cepas MDR y XDR, mayores costos y mortalidad, que necesitarán a su vez un mayor gasto que el que se ha pretendido ahorrar o redirigir. El abandono financiero de los programas de control de la TB ha resultado en una reemergencia de la enfermedad, de la mortalidad, de cepas resistentes y finalmente de mayores presupuestos ${ }^{12,13,19,47,59-62}$. La disminución del presupuesto central del PROCET contrasta con la expansión del presupuesto global de salud.

La presión efectuada por la OPS y algunas sociedades científicas, permitieron una reasignación de recursos que empezó a funcionar desde el año 
2015. Aunque se ha logrado recuperar el presupuesto, lo que ha favorecido la modernización de los laboratorios con un diagnóstico molecular a nivel regional a escala nacional desde el 2017 y el estudio de susceptibilidad universal desde el 2014, la dotación de RRHH sigue siendo deficitaria.

En conclusión, ha habido un grave deterioro de la situación epidemiológica de la TB en Chile que sucede en varias regiones, está asociada al crecimiento porcentual de varios grupos de riesgo y ocurre en conjunto con recortes financieros, problemas operativos y escasez de RRHH. Los avances observados en técnicas diagnósticas y estudio de susceptibilidad han sido notables y permitirán avances en el control y tratamiento de la TB en Chile. Sin embargo, de no mediar un refuerzo de los RRHH especialmente locales para mejorar las numerosas actividades del Programa en forma focalizada, existe un gran riesgo de que el deterioro actual se transforme en franca reemergencia.

\section{Referencias}

1. Borgdorff MW, Floyd K, Broekmans JF. Interventions to reduce tuberculosis mortality and transmission in low-and middle-income countries. Bull WHO 2002; 80 (3): 217-22.

2. Chan-Yeung M, Yeh AG, Tam CM, Kam KM, Leung CC, Yew WW, Lam CW. Socio-demographic and geographic indicators and distribution of tuberculosis in Hong Kong: a spatial analysis. Int J Tuberc Lung Dis 2005; 9 (12): 1320-6.

3. Low CT, Lai PC, Tse WS, Tsui CK, Lee H, Hui PK. Exploring tuberculosis by types of housing development. Soc Sci Med 2013; 87: 77-83.

4. Bhargava A, Pai M, Bhargava M, Marais BJ, Menzies D. Can social interventions prevent tuberculosis? The $\mathrm{Pa}$ pworth experiment (1918-1943) revisited. Am J Respir Crit Care Med 2012; 186 (5): 442-9.

5. Couceiro L, Santana P, Nunes C. Pulmonary tuberculosis and risk factors in Portugal: a spatial analysis. Int J Tuberc Lung Dis 2011; 15 (11): 1445-54.

6. Canadian Tuberculosis Committee. Housing conditions that serve as risk factors for tuberculosis infection and disease. An Advisory Committee Statement (ACS). Can Commun Dis Rep 2007; 33 (ACS-9): 1-13.

7. Beggs CB, Noakes CJ, Sleigh PA, Fletcher LA, Siddigi K. The transmission of tuberculosis in confined spaces: An analytical review of alternative epidemiological models. Int J Tuberc Lung Dis 2003; 7 (11): 1015-26.
8. Lienhardt C. From exposure to disease: The role of environmental factors in susceptibility to and development of tuberculosis. Epidemiol Rev 2001; 23 (2): 288-301.

9. Paluzzi JE. A social disease/a social response: lessons in tuberculosis from early 20th century Chile. Soc Sci Med 2004; 59 (4): 763-73.

10. Akachi Y, Zumla A, Atun R. Investing in improving performance of national tuberculosis programs reduces the tuberculosis burden: Analysis of 22 high-burden countries, 2002-2009. J Infect Dis 2012; 205 Suppl 2: S284-92.

11. Chapple W, Katz AR, Li D. Association between national tuberculosis program budgets and tuberculosis outcomes: an ecological study. Pan African Med J 2012; 12: 66.

12. Freudenberg N, Fahs M, Galea S, Greenberg A. The impact of New York City's 1975 fiscal crisis on the tuberculosis, HIV, and homicide syndemic. Am J Public Health 2006; 96 (3): 424-34.

13. Fica A, Cabello F. Cólera en Latinoamérica: La relación entre salud pública y economía. Interciencia 1992; 17: 275-83.

14. Raviglione MC, Snider DE Jr, Kochi A. Global epidemiology of tuberculosis. Morbidity and mortality of a worldwide epidemic. JAMA 1995; 273 (3): 220-6.

15. MINSAL, Chile. Tuberculosis. Informe de situación 2012. Disponible en: http://web.minsal.cl/sites/default/ files/files/tuberculosisInforme2012.pdf [Consultado el 26 de enero del 2018].

16. Yáñez A. Tuberculosis en inmigrantes. Situación Chile-Perú. Rev Chil Enferm Respir 2010; 26 (3): 161-64.

17. United Nations Development Programme. Briefing note for countries on the 2016 Human Development Report 2016. Disponible en http://hdr.undp.org/sites/all/themes/hdr_theme/country-notes/CHL.pdf [Consultado el 5 de diciembre de 2017].

18. Romero H, Vildosola J. Control of tuberculosis in Chile, 1952. Am J Public Health Nations Health 1953; 43 (6 Pt 1): 724-7.

19. Alarcón A. Plan reducción tuberculosis en el Perú. Disponible en t: http://www.parsalud.gob.pe/phocadownload/plan/plan11.pdf [Consultado el 25 de enero de 2018].

20. MINSA, Perú. Situación de la tuberculosis en el Perú. Disponible en: http://www.minsa.gob.pe/portada/Especiales/2011/respiravida/archivos/Ayuda_memoria_Lanzamiento_TB.pdf [Consultado el 26 de enero de 2018].

21. OPS. Tuberculosis in the Americas: Regional Report 2012. Epidemiology, Control and Financing. Washington, DC: OPS, 2013. Disponible en: http://www. paho.org/hq/index.php?option=com_docman\&task=- 
doc_view\&gid=22953\&Itemid $=[$ Consultado el 30 de julio de 2018].

22. OPS. Situación de la tuberculosis en las Américas, 2015. Disponible en: http://www.paho.org/hq/index. php?option $=$ com_docman\&task=doc_download\&Itemid $=270 \&$ gid $=36278 \&$ lang $=$ es $[$ Consultado el $30 \mathrm{de}$ julio de 2018].

23. USAID. Diagnóstico de la situación de la tuberculosis en 16 municipios priorizados de Bolivia. Disponible en: https://www.usaidassist.org/sites/assist/files/bolivia_tb_rapidassessment.pdf [Consultado el 26 de enero de 2018].

24. MINSAL, Chile. Tuberculosis. Informe de situación 2016. Disponible en: http://diprece.minsal.cl/wrdprss_minsal/wp-content/uploads/2017/07/2017.07.04_Infrome-Tuberculosis.pdf [Consultado el 30 de marzo de 2018].

25. MINSAL, Chile. Tuberculosis. Informe de situación 2008-2011. Disponible en: http://web.minsal.cl/sites/ default/files/files/TB2008-2011.pdf [Consultado el 30 de mayo de 2018]

26. MINSAL, Chile. Tuberculosis. Informe de situación 2013. Disponible en: http://web.minsal.cl/sites/default/ files/files/TUBERCULOSIS-INFORME\%202013.pdf [Consultado el 30 de mayo de 2018].

27. MINSAL, Chile. Tuberculosis. Informe de situación 2017. Disponible en: https://diprece.minsal.cl/wp-content/uploads/2018/10/2018.10.05_TUBERCULOSIS-2017.pdf [Consultado el 15 de junio del 2019].

28. Ministerio de Desarrollo Social. Casen 2013 (Encuesta de Caracterización Socioeconómica nacional). Disponible en: http://observatorio.ministeriodesarrollosocial.gob.cl/documentos/Presentacion_Resultados_Encuesta_Casen_2013.pdf [Consultado el 26 de enero de 2018].

29. Ministerio de Desarrollo Social, Chile. Informe de Desarrollo Social 2014. Disponible en: http://www.ministeriodesarrollosocial.gob.cl/pdf/upload/IDS_2014_2. pdf [Consultado el 15 de marzo del 2018].

30. Ministerio de Desarrollo Social, Chile. Informe de Desarrollo Social 2016. Disponible en: http://www. ministeriodesarrollosocial.gob.cl/pdf/upload/IDS2016. pdf [Consultado el 15 de marzo del 2018].

31. MINSAL, Chile. Tuberculosis. Informe de situación, Chile 2014. Disponible en: http://web.minsal.cl/sites/ default/files/Informe_tbc_2014.pdf [Consultado el 24 de enero del 2018].

32. MINSAL, Chile. Tuberculosis. Informe de situación, Chile 2015. Disponible en: http://diprece.minsal.cl/wrdprss_minsal/wp-content/uploads/2016/08/TUBERCULOSIS-2015.pdf [Consultado el 24 de enero de 2018].
33. Rojas N, Silva C. La migración en Chile: Breve reporte y caracterización. Informe Observatorio Iberoamericano sobre Movilidad Humana, Migraciones y Desarrollo. Disponible en http://www.extranjeria.gob.cl/media/2016/08/informe_julio_agosto_2016.pdf [Consultado el 2 de diciembre de 2017].

34. Ministerio de Desarrollo Social. Casen 2013. Inmigrantes, Síntesis de resultados. Disponible en: http://observatorio.ministeriodesarrollosocial.gob.cl/documentos/ CASEN_2013_Inmigrantes_revisada.pdf [Consultado el 15 de marzo de 2018].

35. Joint United Nations Programme on HIV/AIDS (UNAIDS). Ending AIDS Progress towards the 90-9090 targets. GLOBAL AIDS UPDATE, 2017. Disponible en: http://www.unaids.org/sites/default/files/media_asset/Global_AIDS_update_2017_en.pdf. [Consultado el 26 de enero de 2018].

36. Instituto de Salud Pública. Resultados confirmación de infección por VIH en Chile, 2010-2015. Boletín Vigilancia de laboratorio 2016; 6. Disponible en: http://www. ispch.cl/sites/default/files/BoletinVIH-15112017A.pdf [Consultado el 26 de enero de 2018]

37. Beltrán C, Zitko P, Wolff M, Bernal F, Asenjo A, Fernández AM, et al. Evolución de las características epidemiológicas y clínicas de pacientes adultos del programa nacional al inicio de la terapia anti-retroviral en la Cohorte Chilena de SIDA, 2001-2015. Rev Chilena Infectol 2016; 33 (Supl 1): 2-10.

38. Wolff M, Beltrán C. Programa de acceso ampliado a terapia anti retroviral en Chile, Plan AUGE y Cohorte chilena de SIDA: tres iniciativas complementarias. Rev Chilena Infectol 2004; 21 (4): 281-4.

39. Stuardo V. La prevención olvidada, la reemergencia del VIH en Chile. Rev Chilena Infectol 2017;34 (4):419-20.

40. Riquelme MC, Velasco M, Rodríguez L. Actualización de la resistencia a drogas antituberculosas en Chile, 2006. Rev Chil Enfer Respir 2008; 24 (1): 60-5.

41. Zúñiga M. La eliminación de la tuberculosis como problema de salud pública. Situación de Chile en el año 2008. Rev Chil Enf Respir 2009; 25 (2): 117-26.

42. Herrera T. La situación de la tuberculosis en Chile y los actuales desafíos. Visita de la OPS al programa de control de la tuberculosis en Chile. Rev Chil Enf Respir 2013; 29 (1): 46-9.

43. Secretaría Regional Ministerial, MINSAL, Chile. Resolución exenta 1515 sobre el sumario sanitario 3739 año 2014, 9 de Febrero, 2015.

44. Peña C, Farga V. El difícil camino del control sanitario de la tuberculosis . Rev Chil Enf Respir 2012; 28 (4): 311-8.

45. González C. Cobertura de vacunas. Desafíos para el 
control de enfermedades inmunoprevenibles. VI curso Actualización de Vacunas organizado por la Sociedad Chilena de Infectología. Disponible en: http://www. sochinf.cl/documentos/2014/pre_vacunas/5_Dra_Gonzalez.pdf [Consultado el 25 de enero de 2018].

46. Balcells ME, García P, Chanqueo L, Bahamonde L, Lasso M, Gallardo AM, et al. Rapid molecular detection of pulmonary tuberculosis in HIV-infected patients in Santiago, Chile. Int J Tuberc Lung Dis 2012; 16 (10): 1349-53.

47. Cabello F. Salud pública e ideología: A propósito de las relaciones entre las pandemias de tuberculosis y del SIDA. Salud y Cambio 1994; 5: 19-36.

48. Centers for Disease Control and Prevention. Tuberculosis trends-United States, 2014. MMWR Morb Mortal Wkly Rep 2015; 64 (10): 265-85.

49. Dara M, de Colombani P, Petrova-Benedict M, Centis R, Zellweger JP, Sandgren A, et al. Minimum package for cross-border TB control and care in the WHO European region: a Wolfheze consensus statement. Eur Respir J 2012; 40 (5): 1081-90.

50. Kruishjaar ME, Abubakar I, Stagg HR, Pedrazzoli D, Lipman M. Migration and tuberculosis in UK: targeting screening for latent infection to those at greatest risk of disease. Thorax 2013; 68 (12): 1172-74.

51. Tankimovich M. Barriers to and interventions for improved tuberculosis detection and treatment among homeless and immigrant populations: A literature review. J Community Health Nurs 2013; 30 (2): 83-95.

52. García C. Tuberculosis en grupos de riesgo en la Región Metropolitana, 2008. Rev Chil Enf Respir 2010; 26 (2): 105-11.

53. Aguilera X, González C, Nájera M, Hirmas M, Delgado $\mathrm{I}$, Olea A, et al. Tuberculosis in prisoners and their contacts in Chile: estimating incidence and latent infection. Int J Tuberc Lung Dis 2016; 20 (1): 63-30.

54. Kearney MS, Harris BH, Jácome E, Parker L. Ten economic facts about crime and incarceration in the United States. The Hamilton Project. Policy Memo May
2014. Disponible en: http://www.hamiltonproject.org/ files/downloads_and_links/v8_THP_10CrimeFacts.pdf [Consultado el 10 de marzo de 2018].

55. Carranza E. Situación penitenciaria en América Latina y el Caribe ¿Qué hacer? Anuario de derechos humanos 2012:31-64. Disponible en http://www.anuariocdh. uchile.cl/index.php/ADH/article/viewFile/20551/21723 [Consultado el 26 de enero de 2018].

56. Instituto Nacional de Derechos Humanos. Estudio de las condiciones carcelarias en Chile. Diagnóstico del cumplimiento de los estándares internacionales de los derechos humanos. 2013. Disponible en: http:// www.pazciudadana.cl/wp-content/uploads/2014/03/ m15861-estudio-de-las-condiciones-carcelarias.pdf [Consultado el 26 de enero de 2018].

57. Biadglegne F, Rodloff AC, Sack U. Review of the prevalence and drug resistance of tuberculosis in prisons: a hidden epidemic. Epidemiol Infect 2015; 143 (5): 887900.

58. Sacchi FP, Praça RM, Tatara MB, Simonsen V, Ferrazoli L, Croda MG, et al. Prisons as reservoir for community transmission of tuberculosis, Brazil. Emerg Infect Dis 2015; 21 (3): 452-5.

59. Kim DH, Kim HJ, Park SK, Kong SJ, Kim YS, Kim TH, et al. Treatment outcomes and survival based on drug resistance patterns in multidrug-resistant tuberculosis. Am J Respir Crit Care Med 2010; 182 (1): 113-9.

60. Sidel VW, Drucker E, Martin SC. The resurgence of tuberculosis in the United States: societal origins and societal responses. J Law Med Ethics 1993; 21 (3-4): 303-16.

61. Pooran A, Pieterson E, Davids M, Theron G, Dheda K. What is the cost of diagnosis and management of drug resistant tuberculosis in South Africa? Plos One 2013; 8 (1): e54587.

62. Dukes Hamilton C, Sterling TR, Blumberg HM, Leonard M, McAuley J, Schlossberg D, et al. Extensively drug-resistant tuberculosis: are we learning from history or repeating it? Clin Infect Dis 2007; 45 (3): 338-42. 